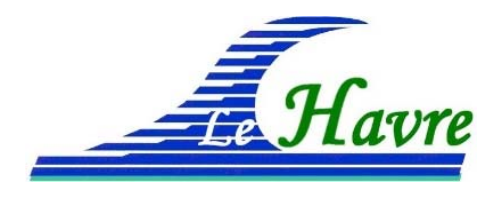

XVI'̀mes Journées Nationales Génie Côtier - Génie Civil

Le Havre, 2020

DOI:10.5150/jngcgc.2020.012 (C) Editions Paralia CFL

disponible en ligne - http://www.paralia.fr - available online

\title{
Adaptation d'un modèle de circulation océanique à la modélisation déterministe de la houle : application à la plage de Sète
}

\author{
Patrick MARSALEIX ${ }^{1}$, Héloïse MICHAUD ${ }^{2}$, Léo SEYFRIED ${ }^{2}$ \\ 1. LEGOS, UMR5566, Université de Toulouse, CNRS, 14 avenue Edouard Belin, 31400 \\ Toulouse, France \\ patrick.marsaleix@legos.obs-mip.fr \\ 2. Shom, Antenne de Toulouse, BP 45017 - 31032 Toulouse, France \\ heloise.michaud@shom.fr; leo.seyfried@shom.fr
}

\section{Résumé :}

Le modèle de circulation océanique côtière SYMPHONIE a été adapté à la modélisation déterministe de la houle. Ses équations, désormais non-hydrostatiques, s'écartent de la formulation exacte associée à l'hypothèse d'incompressibilité afin de réduire le coût du calcul. Dans une précédente étude (MARSALEIX et al, 2019), les auteurs discutent de la légitimité de leurs hypothèses et testent leur modèle sur des expériences académiques en laboratoire. On rappelle les principaux points de cette précédente étude et la préparation d'une application à un cas réaliste, en l'occurrence la plage de Sète, sur une période caractérisée par de fortes vagues.

\section{Mots-clés :}

Modèle SYMPHONIE, Modélisation déterministe de la houle.

\section{Introduction}

Le modèle SYMPHONIE est un modèle de circulation océanique aux échelles régionale et côtière (ESTOURNEL et al., 2012 ; ESTOURNEL et al., 2016) récemment adapté à la modélisation déterministe de la houle (MARSALEIX et al., 2019, par la suite abrégé MME19). Cette adaptation préserve les aptitudes du modèle d'origine à représenter la circulation océanique et permet donc l'étude des interactions de la houle et des courants basse fréquence selon une approche entièrement déterministe. Ces interactions, tridimensionnelles, peuvent s'étendre aux autres champs du modèle, à savoir la température, la salinité, la fermeture turbulente. L'adaptation du modèle à la simulation déterministe de la houle a principalement concerné l'implémentation de la pression nonhydrostatique dans des équations faisant jusqu'alors l'hypothèse de l'équilibre hydrostatique (MARSALEIX et al., 2008). Les nouvelles équations s'écartent toutefois de la formulation exacte dictée par l'hypothèse d'incompressibilité afin de réduire le coût du calcul. Dans leur précédente étude, MME19 discutent de la légitimité de leurs hypothèses. Les principaux éléments de cette discussion sont rappelés dans le paragraphe 2. Jusqu'ici la version "houle déterministe" de SYMPHONIE a été validée par MME19 


\section{Thème 1 - Hydrodynamique côtière}

sur des expériences en laboratoire de propagation de vagues sur des plages idéalisées (HAMILTON, 2001), incluant éventuellement une barre topographique favorisant le déferlement et indentée de passages favorisant le développement de courants sagittaux (HALLER et al., 2002). Dans la présente étude, l'évaluation des capacités du modèle est étendue à un cas réaliste, en l'occurrence la plage de Sète. La période considérée est début février 2009 caractérisé par un événement de vent fort en provenance de sud-est. Les conditions aux limites sur la frontière ouverte au large sont fournies par une simulation de vagues produite par un modèle spectral implémenté sur un domaine de plus grande emprise.

\section{Description du modèle}

\subsection{Equations}

On rappelle ici seulement les principaux enjeux des équations non-hydrostatiques de notre modèle et à cette fin les équations présentées en suivant ne retiennent que les termes essentiels à notre propos et ce dans un plan bidimensionnel vertical (pour une description complète et détaillée voir MME19). Dans ce même cadre restreint on commence par rappeler la formulation usuelle du problème dans le cadre de l'hypothèse d'incompressibilité :

$\frac{\partial u}{\partial t}=-g \frac{\partial \eta}{\partial x}-\frac{\partial q}{\partial x}$

$\frac{\partial \eta}{\partial t}=-\frac{\partial}{\partial x} \int_{-h}^{\eta} u d z$

$0=g \frac{\partial^{2} \eta}{\partial x^{2}}+\frac{\partial^{2} q}{\partial x^{2}}+\frac{\partial^{2} q}{\partial z^{2}}$

où : g est la gravité, $u$ la composante horizontale du courant, $\eta$ la hauteur de la surface, $q$ la pression dynamique, $h$ la bathymétrie. Le courant horizontal et la hauteur de la surface sont calculés itérativement par les équations (1) et (2) et la pression dynamique est donnée par la résolution du problème de poisson exprimé par l'équation (3). Bien qu'en soit inutile à la résolution du problème tel que nous venons de le formuler il est possible de déduire la vitesse verticale, $w$, en évoquant l'hypothèse d'incompressibilité:

$\frac{\partial u}{\partial x}+\frac{\partial w}{\partial z}=0$

La solution au problème de Poisson (3) passe normalement par la résolution d'un système d'équations linéaires dont le coût numérique est en principe important. L'appréciation de ce coût pouvant varier d'une étude à une autre (selon les performances des méthodes numériques mises en œuvre), nous nous bornerons à dire que dans notre cas nous l'avons jugé prohibitif. Pour dépasser ce point bloquant, nous avons adopté la formulation alternative suivante :

$\frac{\partial u}{\partial t}=-g \frac{\partial \eta}{\partial x}-r \frac{\partial q}{\partial x}$

$\frac{1}{\alpha^{2}} \frac{\partial^{2} q}{\partial t^{2}}=g \frac{\partial^{2} \eta}{\partial x^{2}}+\frac{\partial^{2} q}{\partial x^{2}}+\frac{\partial^{2} q}{\partial z^{2}}$ 


\section{XVI'̀mes Journées Nationales Génie Côtier - Génie Civil \\ Le Havre 2020}

où l'équation de Poisson est maintenant remplacée par (6) qui a la forme générale d'une équation de propagation d'onde où $\alpha$ est une constante homogène à une célérité. Cette dernière est déterminante dans la rapidité de l'ajustement de la pression dynamique aux autres champs, plus grand étant $\alpha$ plus rapide étant l'ajustement (le cas particulier d'une valeur infinie étant équivalent à l'ajustement instantané de la formulation incompressible usuelle). On note ensuite, au second terme du membre de droite de (5), l'apparition de $r$ qui joue le rôle d'un atténuateur du gradient de pression dynamique. L'élévation de la surface continue d'être calculée par (2). Enfin comme précédemment une vitesse verticale peut être déduite de (4). La réduction du coût numérique qu'entraîne cette nouvelle formulation provient de ce que la résolution de (6) est directe, locale et évite par conséquent le calcul d'un système d'équations linéaires. En contrepartie, l'écart à la formulation rigoureuse (1)-(3) entraîne une erreur sur le calcul de la pression dynamique (et en conséquence sur les autres champs également). Le choix de $\alpha$ est en fait au cœur d'un compromis entre le coût du calcul et sa précision, car si augmenter $\alpha$ accroît la précision cela contraint aussi à réduire le pas de temps du schéma forward-backward (ce qui augmente donc le coût de la simulation). Pour un intervalle de bathymétrie typique des applications envisagées avec ce modèle $(0<h<50 \mathrm{~m})$ et des périodes de vagues comprises entre 6 et 14 secondes, MME19 montrent qu'une précision acceptable peut être obtenue pour $\alpha$ de l'ordre de 4 fois la vitesse de phase des ondes longues (soit $c \simeq 4 \sqrt{g h}$ ) pour peu qu'une valeur appropriée de $r$ soit utilisée. Cette dernière peut être déterminée soit de manière empirique en s'appuyant sur une simulation de référence considérée comme "exacte" (c.à.d. s'appuyant sur une valeur suffisamment grande de $\alpha$ ) soit par un raisonnement fondé sur les solutions analytiques au problème de la propagation des vagues sur un fond plat dans le cadre de l'approximation linéaire. D'une part on rappelle que la relation de dispersion associée aux équations (1)-(3) est :

$\omega^{2}=g k \tanh (k h)$

où : $k$ est le vecteur d'onde et $\omega$ la fréquence angulaire. D'autre part dans MME19 on montre que la relation de dispersion associée à (5)-(6) est :

$\omega^{2}=k^{2} g\left(1-\frac{r}{\mu^{2}}\right) h+\frac{r}{\mu^{3}} g k \tanh (\mu k h)$

où :

$\mu^{2}=\left(1-\frac{\omega^{2}}{k^{2} \alpha^{2}}\right)$

On note tout d'abord que dans le cas particulier $r=1$ et $\alpha=\infty$ les deux relations de dispersions (7) et (9) sont équivalentes, comme attendu. Dans le cas où, comme évoqué plus haut, $\alpha$ est de l'ordre de 4 fois la vitesse de phase des ondes longues, MME19 montrent que l'erreur relative sur la longueur d'onde (pour une période donnée dans l'intervalle $6 \mathrm{~s}$ à $14 \mathrm{~s}$ susmentionné) est de l'ordre de quelques \%. Le paramètre $r$ peut être alors utilisé pour réduire l'erreur en choisissant une valeur rendant équivalente les expressions (7) et (9) pour une période des vagues choisie (celle correspondant aux vagues dominantes), ce qui donne : 


\section{Thème 1 - Hydrodynamique côtière}

$r=\mu^{3} \frac{\tanh (k h)-k h}{\tanh (\mu k h)-\mu k h}$

Les valeurs produites par (10) sont légèrement inférieures à 1 (0.97 est une valeur typique) d'où l'expression employée précédemment d'atténuateur du gradient de pression dynamique. D'autre part MME19 montrent qu'annuler l'erreur pour une période donnée réduit considérablement l'erreur pour les périodes avoisinantes et qu'au final, il est possible de réduire l'erreur relative à moins de $1 \%$ sur l'ensemble du spectre de vagues considéré et enfin qu'améliorer les propriétés de dispersion via (10) améliore également les autres champs du modèle, notamment le courant.

\subsection{Les conditions aux limites}

Les conditions aux limites latérales ouvertes ont deux fonctions : une fonction "active" pour introduire les vagues venant du large et une fonction "passive" pour laisser passer les vagues sortantes en limitant le plus possible les artéfacts numériques conduisant à des réflexions artificielles. Dans la présente application, la grille numérique est de forme rectangulaire, le côté le plus long étant globalement parallèle à la ligne de côte dans sa partie rectiligne au Sud de Sète (Figure 1). Une des frontières latérales est donc entièrement "fermée". En vis à vis, la frontière latérale ouverte au large est celle par laquelle entrent les vagues venant du large. Les deux frontières latérales restantes sont plus courtes, grosso modo perpendiculaires à la ligne de côte, et leur unique fonction est de laisser passer les vagues sortant du domaine numérique. La frontière ouverte au large doit en revanche pouvoir fonctionner simultanément de manière active et passive. A cette fin, les champs des vagues sortantes ( $\left.\eta^{\prime}, q^{\prime}, u^{\prime}\right)$ sont tout d'abord définis comme étant la différence entre les champs totaux et les champs entrant (BLAYO \& DEBREU, 2005). En pratique on introduit donc $\eta^{\prime}=\eta-\eta_{w}, q^{\prime}=q-q_{w}, u^{\prime}=u-u_{w}$ où $\left(\eta_{w}, q_{w}, u_{w}\right)$ sont les champs des vagues entrantes, déduites d'une simulation d'un modèle spectral de vagues de plus grande emprise. La condition aux limites de Flather (FLATHER, 1976) est ensuite étendue au cas des ondes courtes :

$u^{\prime}= \pm \frac{g \eta^{\prime}+q^{\prime}}{c}$

où le choix du signe au membre de droite dépend de l'orientation locale de l'axe numérique normal à la frontière et où $c$ est une vitesse de phase choisie de manière empirique (dans le cas particulier d'une propagation perpendiculaire à la frontière, idéalement celle-ci serait donnée par la théorie linéaire correspondant à la fréquence supposée de ces vagues). Les champs fournis par le modèle spectral sont transformés en champs déterministes à partir de la théorie linéaire. L'élévation de la surface est donnée par :

$\eta_{w}(x, y)=\sum_{\omega, \theta} \sqrt{2 E_{\omega, \theta}(x, y)} \cos \left(\omega t+\varphi_{\omega, \theta}(x, y)\right) \Delta \omega \Delta \theta$

où : $E_{\omega, \theta}$ est la densité fréquentielle et directionnelle de la variance de l'élévation de l'élévation de la surface autour de la fréquence $\omega$ et de la direction $\theta$, calculée par le 


\section{XVIèmes Journées Nationales Génie Côtier - Génie Civil \\ Le Havre 2020}

modèle WW3 (WW3DG, 2016). La phase $\varphi_{\omega, \theta}$ est déduite de $\left(k_{\omega, \theta}^{x}, k_{\omega, \theta}^{y}\right)$ les composantes du vecteur d'onde :

$\left(\frac{\partial \varphi_{\omega, \theta}(x, y)}{\partial x}, \frac{\partial \varphi_{\omega, \theta}(x, y)}{\partial y}\right)=\left(k_{\omega, \theta}^{x}(x, y), k_{\omega, \theta}^{y}(x, y)\right)$,

le module du vecteur d'onde étant déduit de la fréquence, de la bathymétrie au point $(x, y)$ considéré et de la relation de dispersion (7). On note que la phase est en fait déduite de (13) à une constante près que l'on détermine arbitrairement à partir d'une fonction aléatoire. $\Delta \omega$ et $\Delta \theta$ correspondent à la résolution fréquentielle et directionnelle du modèle spectral qui s'appuie sur 30 fréquences discrètes (périodes de 1s à 16s) et 24 directions discrètes. Notre modélisation déterministe ne retient d'une part que les périodes supérieures à 5 secondes (les longueurs d'ondes des autres périodes étant considérées trop petites pour la résolution horizontale de notre grille) et d'autre part que les 12 directions correspondant aux vagues pénétrant dans le domaine par sa frontière latérale ouverte au large. Par un raisonnement analogue à (13) et en utilisant les formulations du courant et de la pression dynamique de la théorie linéaire (KINSMAN, 1965), on obtient $\left(q_{w}, u_{w}\right)$, les autres champs requis par la condition aux limites.

\subsection{Déferlement, swash}

Nous signalons tout d'abord que, dans le souci de simplifier leur écriture, les formules données en suivant reprennent le formalisme simplifié $O x z$ adopté pour les équations (1) à (6). La paramétrisation du déferlement distingue deux types de situations. Celle dont l'occurrence est la plus grande concerne le déferlement consécutif à une amplification critique de la vague détectée à partir de $f_{b}$ une métrique de type "nombre de Froude" mesurant l'importance relative du courant du surface par rapport à la vitesse de phase:

$f_{b}=c_{1}\left[\frac{u_{s}^{2}+\left(k^{-1} \frac{\partial u_{s}}{\partial x}\right)^{2}}{g h}\right]$

où $u_{s}$ est le courant de surface et $c_{1}$ une constante de calibration. Au dénominateur de (14), la vitesse de phase (au carré) est approximée à la vitesse des ondes longues (c.a.d. $\sqrt{g h}$ ) considérée légitime en eaux peu profondes (où l'on fait l'hypothèse que se produit l'essentiel du déferlement). En pratique $f_{b}$ est une grandeur sans unité, proche de sa valeur maximale lorsque le courant de surface approche la vitesse de phase et proche de zéro dans le cas contraire. On définit par ailleurs une échelle homogène à un coefficient de viscosité horizontal "moyen" qui s'écrit :

$<v_{b}>=\frac{3}{8} g^{3 / 2} h^{1 / 2}\left|\frac{\partial h}{\partial x}\right|\left(\frac{T}{\pi}\right)^{2}$

où : $T$ est la période dominante des vagues. Les arguments et les détails des calculs conduisant à (14) et (15) sont données par MME19. En pratique le produit $f_{b}\left\langle v_{b}\right\rangle$ donne le coefficient de viscosité horizontale d'un opérateur de type Laplacien appliqué à la vitesse. Cet opérateur conserve la quantité de mouvement totale ce qui signifie que la quantité de mouvement localement perdue par les vagues par ce procédé est transmise 


\section{Thème 1 - Hydrodynamique côtière}

intégralement aux plus grandes échelles d'espace et de temps. La seconde situation de déferlement envisagée dans notre modélisation est celle des vagues se brisant sur les enrochements bordant les digues des installations portuaires. La présence de ces enrochements est paramétrée sous forme d'une augmentation locale de la friction, en l'occurrence l'augmentation de $z_{0}$ la longueur de la rugosité de fond intervenant dans le formalisme adopté pour la tension du fond. Par défaut égal à $10^{-4} \mathrm{~m}$ en dehors des zones d'enrochement, $z_{0}$ atteint quelques dizaines de centimètres en bordure des digues. Hors de la zone portuaire et de ses enrochements, la ligne de côte de notre de domaine est constituée de plages sableuses faiblement inclinées où le swash (et plus généralement la submersion) est modélisé grâce au schéma de wetting drying 3D du modèle.

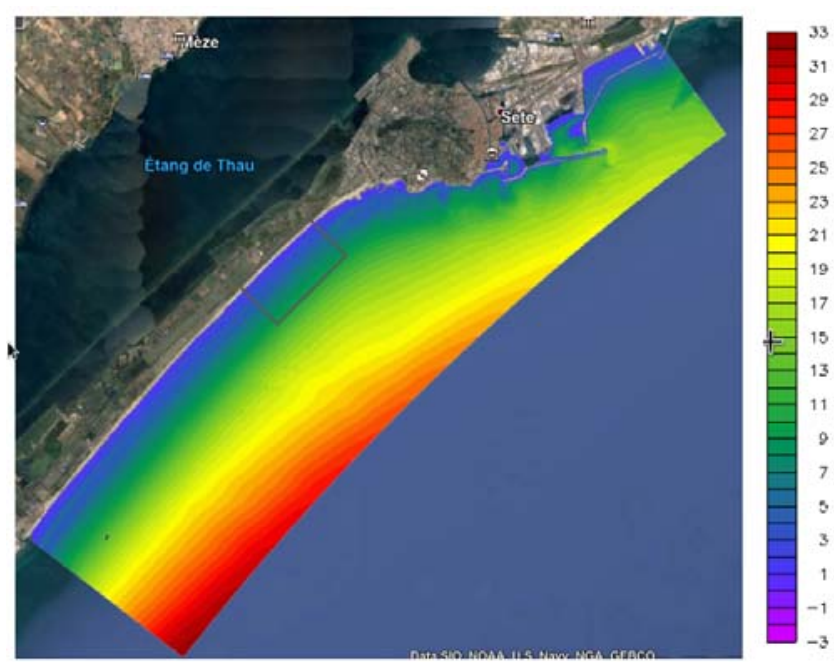

Figure 1. Bathymétrie (m) du domaine numérique. Le rectangle noir correspond à la zone couverte par la figure 2.

\section{Application à la plage de Sète}

Le domaine numérique (figure 1) s'étend sur environ $18 \mathrm{~km}$ par $4.7 \mathrm{~km}$ avec une grille 3D structurée de type $\mathrm{C}$, contenant 4500 points dans la direction parallèle à la direction principale de la ligne de côte, 1500 points dans la direction perpendiculaire à la côte et, sur la verticale, 10 points répartis entre le fond et la surface selon le principe de la coordonnée sigma. La résolution horizontale est de $4 \mathrm{~m}$ dans la direction parallèle à la côte. Dans la direction perpendiculaire à la côte la résolution, variable, est comprise entre $4 \mathrm{~m}$, au large, et $2 \mathrm{~m}$ à la côte. Le pas de temps est de 0.027 secondes. La bathymétrie correspond à une campagne lidar de 2011 sur une grille régulière de $5 \mathrm{~m}$ de résolution. La période choisie correspond à début février 2009 en raison des conditions météorologiques favorables à des vagues de fortes amplitudes autour de la période $8 \mathrm{~s}$. 


\section{XVI'̀mes Journées Nationales Génie Côtier - Génie Civil}

Le Havre 2020

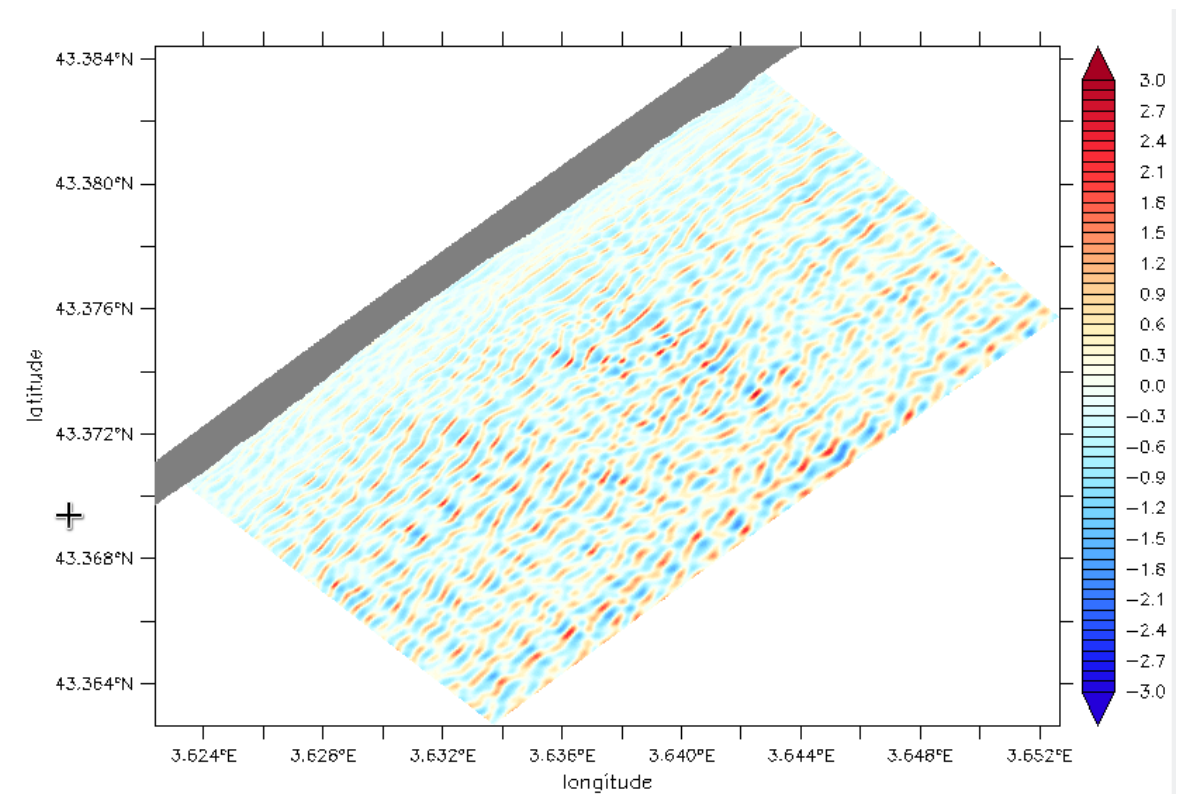

Figure 2. Elévation de la surface (m) instantanée sur une portion domaine correspondant au rectangle noir de la figure 1.

\section{Conclusions}

L'étude en est actuellement au stade préliminaire de la mise en place du modèle et de l'analyse des premiers résultats (figure 2). Si les hypothèses faites par le modèle pour son approche non-hydrostatique semblent jusqu'alors robustes, la question de la paramétrisation du déferlement reste encore en suspens et constitue une priorité pour les auteurs. Compte tenu de cet objectif, et dans la perspective des JNGCGC au Havre en juin 2020, l'étude sera étendue à des comparaisons à des mesures in situ de la hauteur significative des vagues à différentes distances de la côte dans le voisinage des barres sableuses, autrement dit à différents stades du déferlement des vagues.

\section{Références bibliographiques}

AUCLAIR F. (2012). Assessment of the amount of Cesium-137 released into the Pacific Ocean after the Fukushima accident and analysis of its dispersion in Japanese coastal waters. Journal of Geophysical Research, Vol. 117, C11014, http://dx.doi.org/10.1029/2012JC007933

BLAYO E., DEBREU L. (2005). Revisiting open boundary conditions from the point of view of characteristic variables. Ocean Modeling. Vol. 9, pp 231-252 https://doi.org/10.1016/j.ocemod.2004.07.001

ESTOURNEL C., BOSC E., BOCQUET M., ULSES C., MARSALEIX P., WINIAREK V., OSVATH I., NGUYEN C., DUHAUT T., LYARD F., MICHAUD H., ESTOURNEL C., TESTOR P., DAMIEN P, D'ORTENZIO F., MARSALEIX P., CONAN P., KESSOURI F., DURRIEU DE MADRON X., COPPOLA L., LELLOUCHE J.-M., 


\section{Thème 1 - Hydrodynamique côtière}

BELAMARI S., MORTIER L., ULSES C., BOUIN M.-N., PRIEUR L. (2016). High resolution modeling of dense water formation in the north-western Mediterranean during winter 2012-2013: Processes and budget. Journal of Geophysical Research. http://dx.doi.org/10.1002/2016JC011935

FLATHER R.A. (1976). A tidal model of the Northwest European continental shelf. Mémoires de la Société Royale des Sciences de Liège Vol. 10, pp 141-164.

HALLER M.C., DALRYMPLE R.A., SVENDSEN I.A. (2002). Experimental study of neashore dynalics on a barred beach with rip channels. Journal of Geophysical Research, 107(C6), pp 14.1-14.21. https://doi.org/10.1029/2001JC000955

HAMILTON D.G., EBERSOLE B.A. (2001). Establishing uniform longshore currents in a large scale sediment transport facility. Coastal Engineering, Vol. 42, pp 199-218. https://doi.org/10.1016/S0378-3839(00)00059-4

KINSMAN B. (1965). Wind Waves: Their generation and propagation on the ocean surface. Courier Dover Publications, 676 p.

MARSALEIX P., AUCLAIR F., FLOOR J.W., HERRMANN M.J., ESTOURNEL C., PAIRAUD I., ULSES C. (2008). Energy conservation issues in sigma-coordinate freesurface ocean models. Ocean Modelling, Vol. 20, pp 61-89. http://dx.doi.org/10.1016/j.ocemod.2007.07.005

MARSALEIX P., MICHAUD H., ESTOURNEL C. (2019). 3D phase-resolved wave modelling with a non-hydrostatic ocean circulation model. Ocean Modelling, Vol. 136, pp 28-50. https://doi.org/10.1016/j.ocemod.2019.02.002

WW3DG-The WAVEWATCH III ${ }^{\circledR}$ Development Group. (2016). User manual and system documentation of WAVEWATCH III ${ }^{\circledR}$ version 5.16. Tech. Note 329, NOAA/NWS/NCEP/MMAB, College Park, MD, USA, 326 pp + Appendices. Disponible en ligne sur URL : https://polar.ncep.noaa.gov/waves/wavewatch/manual.v5.16.pdf 